\title{
Current Concepts of Posterolateral Corner Injuries of the Knee
}

\author{
Oog-Jin Shon, MD, Jae-Woo Park, MD, and Beum-Jung Kim, MD \\ Department of Orthopedic Surgery, Yeungnam University Medical Center, Daegu, Korea
}

\begin{abstract}
The number of posterolateral corner (PLC) injury patients has risen owing to the increased motor vehicle accidents and sports activities. Careful examination is required because this injury is easy to overlook and may lead to chronic instability. The purpose of this article is to review the anatomy, biomechanics, diagnosis, classification and, treatment of PLC injuries and summarize the recent literatures regarding the treatment outcomes.
\end{abstract}

Keywords: Knee, Instability, Diagnosis, Treatment

\section{Introduction}

The incidence of posterolateral corner (PLC) injuries has risen owing to the increase in motor vehicle accidents and athletic traumas. PLC injuries are frequently encountered combined with anterior cruciate ligament (ACL) or posterior cruciate ligament (PCL) ruptures, and isolated PLC injuries are rare. However, a PLC injury can be overlooked or misdiagnosed. Left untreated, it can cause chronic pain and residual instability. Thus, it is of utmost importance to detect and treat the injury properly. PLC injuries are not usually diagnosed in the first place, and a thorough understanding of functional interactions of the PLC, specific history taking, and precise physical examination are required for the diagnosis and treatment.

After brief description of the anatomy, biomechanics, and diagnosis of PLC instability, surgical treatments will be described in

Received June 1, 2016; Revised (1st) September 28, 2016;

(2nd) January 4, 2017; (3rd) March 5, 2017

Accepted March 13, 2017

Correspondence to: Oog-Jin Shon, MD

Department of Orthopedic Surgery, Yeungnam University Medical

Center, 170 Hyeonchung-ro, Nam-gu, Daegu 42415, Korea

Tel: +82-53-620-3640, Fax: +82-53-628-4020

E-mail: ossoj@medical.yu.ac.kr

This is an Open Access article distributed under the terms of the Creative Commons Attribution Non-Commercial License (http://creativecommons.org/licenses/by-nc/4.0/) which permits unrestricted non-commercial use, distribution, and reproduction in any medium, provided the original work is properly cited. detail with respect to the methods of surgical reconstruction.

\section{Anatomy}

The anatomically important structures in the PLC of the knee are the lateral collateral ligament (LCL), popliteofibular ligament (PFL), popliteus tendon, popliteofemoral ligament, and posterolateral capsule. The structures are divided into static and dynamic stabilizers. The static stabilizers include the LCL, PFL, arcuate ligament complex, fabellofibular ligament and posterolateral capsule. The dynamic stabilizers are the biceps femoris, iliotibial band (ITB), and popliteus complex (Fig. 1).

The LCL is a static stabilizer that provides restraint against varus forces on the knee. This structure originates just proximal and posterior to the lateral femoral epicondyle and attaches roughly $8.1 \mathrm{~mm}$ posterior to the anterior border of the fibular head. The popliteus complex consists of the popliteus tendon and ligamentous connection. Compared with the LCL, it functions as a dynamic stabilizer on external rotation while the knee is hyperflexed. It inserts approximately $18.5 \mathrm{~mm}$ anterior and distal to the LCL insertion point. The PFL originates from the myotendinous junction of the popliteus muscle and attaches roughly $1.3 \mathrm{~mm}$ distal and $0.5 \mathrm{~mm}$ anterior to the tip of the styloid process of the fibula. It is divided into anterior and posterior sections.

Other structures that contribute to PLC stability are the ITB, biceps femoris, fabellofibular ligament, mid-third of the lateral capsule and lateral meniscus. The ITB is an extension of the 


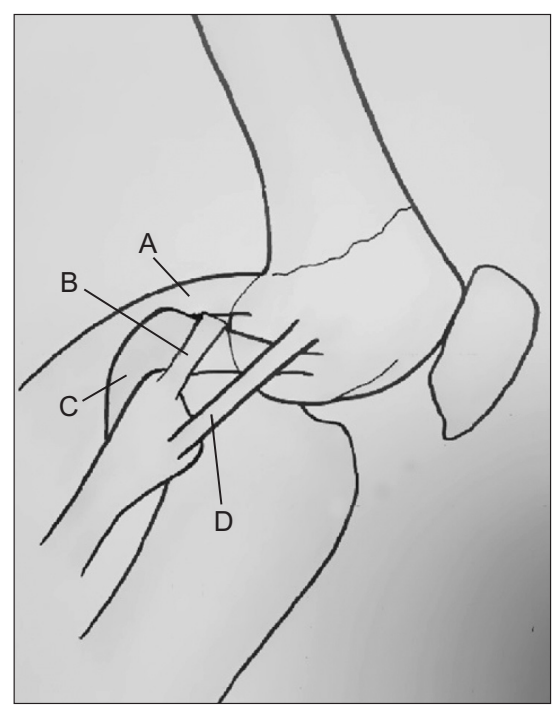

Fig. 1. Anatomy of the posterolateral corner. A: lateral gastrocnemius tendon, B: popliteofibular ligament, C: popliteus muscle and ligament, D: lateral collateral ligament.

fascia over the gluteus maximus and tensor fascia latae muscle. The origins of ITB are the anterior superior iliac spine, anterior border of the ilium, and the external lip of the iliac crest. The insertions of ITB are the lateral intermuscular septum, lateral aspect of the patella and the anterolateral aspect of the lateral tibial plateau at Gerdy tubercle. The ITB provides lateral knee stability when excessive varus stress is loaded in extension of the knee ${ }^{1)}$. The biceps femoris muscle has long and short heads, and the two heads aid the knee with flexion and lateral rotation. It provides dynamic stability at varus angulation, controls tibial internal rotation, and works with the medial hamstrings to prevent excessive tibiofemoral anterior translation. The mid-third lateral capsule plays an important role as a secondary stabilizer to varus stability ${ }^{2}$. The coronary ligament of the lateral meniscus extends from the popliteal hiatus to the popliteomeniscal fascicle and plays a role as a resistance when the knee is in hyperextension or tibial posterolateral rotation ${ }^{3}$. Consequently, the PLC serves as the primary stabilizer that resists varus stress on the knee.

\section{Biomechanics}

The PLC structures provide the primary restraint to varus forces of the knee and also posterolateral rotation of the tibia ${ }^{4)}$. In cruciate deficient knees, these structures play a great role as secondary stabilizers to anterior and posterior tibial translation ${ }^{5,6)}$. Previous biomechanical studies, through selective sectioning of structures, provided evidence on the importance of the LCL, popliteus ten- don, and PFL in resisting forces on the knee ${ }^{5-8)}$.

The LCL is the primary static restraint to varus opening of the knee $^{5-7)}$. Direct force measurements of the LCL during an applied varus movement demonstrate loading responses at all angles of knee flexion, with the response at $30^{\circ}$ of flexion significantly higher than that at $90^{\circ}$ of flexion. The tensile strength of the LCL has been determined to be $295 \mathrm{~N}$. After sectioning of the LCL, LaPrade and Wentorf" also proved that the mean load responses to external rotation in the LCL were significantly higher than those of the popliteus tendon and PFL at $0^{\circ}$ and $30^{\circ}$ of flexion, whereas the popliteus and PFL demonstrated higher loads at higher knee flexions, peaking at $60^{\circ}$. In regards to tibial external rotation, the PLC is the primary stabilizer of external tibial rotation at all knee flexion angles. In studies by both Gollehon et al..$^{5)}$ and Grood et al..$^{6}$, isolated sectioning of the PLC produced a maximal average increase of $13^{\circ}$ of rotation at $30^{\circ}$ of knee flexion, which decreased to an average of $5.3^{\circ}$ at $90^{\circ}$. Conversely, isolated sectioning of the PCL had no effect on external tibial rotation. Combined injury to the PCL and posterolateral structures produced significantly greater increases in external tibial rotation, especially at $90^{\circ}$ of knee flexion $\left(20.9^{\circ}\right)$. Thus, combined PCL and PLC injuries are more susceptible to external rotation forces. The dominant restraint to posterior tibial translation is the PCL. Isolated sectioning of the PCL produces increased posterior tibial translation at all angles of knee flexion, with a maximum at $90^{\circ}$ $(11.4 \mathrm{~mm})$; isolated sectioning of the PLC structures also produces increased posterior tibial translation at all angles of knee flexion, with a maximum at early knee flexion. Therefore, the PLC, not the PCL, is the primary restraint to posterior tibial translation at near full knee extension. Combined sectioning studies of both the PCL and PLC have demonstrated significant increases in posterior translation $(21.5 \mathrm{~mm})$ at $90^{\circ}$ of flexion compared with the intact knee or knees with an isolated PCL injury or posterolateral deficiency. Others have reiterated this strong functional interaction between the popliteus and the PCL; they also have shown how the popliteus acts as both static and dynamic stabilizers of the knee. In a cadaveric study, Harner and Hoher ${ }^{10)}$ found that loading the popliteus in an intact knee reduced in situ forces in the PCL in response to a posterior load, whereas in a PCL-deficient model, loading of the popliteus reduced posterior translation at a maximum of $30^{\circ}$ of knee flexion. Biomechanical analysis of posterolateral deficiency in the setting of ACL or PCL reconstruction further demonstrates the interdependent relationship of the PLC structures and the cruciate ligaments. LaPrade et al. ${ }^{11)}$ noted increased loads in the ACL graft with application of varus and coupled varus-internal rotation moments. Because 
of these significantly increased loads, the authors recommended reconstruction or repair of PLC. The PLC is a minor primary stabilizer (the ACL is the main stabilizer in lower flexion angles and the ALL is in higher flexion angles) in preventing internal rotation. A small, yet significant, increase in internal rotational laxity was demonstrated in a popliteus cutting study ${ }^{11)}$. The other PLC structures are secondary restraints to internal rotation.

Because of the function of PLC, any failure to recognize and treat a PLC injury would result in increased stresses and possible failure in PCL or ACL reconstruction. For this reason, a combined PLC and cruciate ligament reconstruction is recommended $^{12)}$. Similarly, in a combined PCL and PLC injury model by Sekiya et al. ${ }^{13)}$, reconstruction of both structures produced more nearly normal knee kinematics. There has been a recent trend toward more anatomic reconstruction, specifically, of the three most critical biomechanical structures that control varus and external rotation: the LCL, popliteus tendon, and PFL. In a cadaveric study, an anatomic reconstruction demonstrated no significant difference between the intact and reconstructed knees to varus load at $0^{\circ}, 60^{\circ}$, and $90^{\circ}$ of flexion or to external torque at any flexion angle ${ }^{14)}$. However, Some biomechanical studies, in which all three functional components were anatomically reconstructed, separately documented overconstraint of internal rotation and varus rotation, respectively. Yoon et al. ${ }^{15)}$ reported that a recent PLC reconstruction technique that does not reconstruct the dynamic popliteus muscle was not inferior to that involving anatomic reconstruction of the popliteal tendon. Kim et al. ${ }^{16)}$ reported that the 3 established techniques (Warren, Larson, and Kim technique) were not effective in restoring the original strength of the native PLC.

\section{Diagnosis}

\section{History and Injury Mechanism}

\section{1) History}

A thorough history helps to avoid neglecting possible injuries to the PLC of the knee. Pain on the posterolateral aspect of the knee is a typical symptom in the isolated acute PLC injuries. Some patients complain of neurologic symptoms. DeLee et al. ${ }^{17)}$ reported the injury of the peroneal nerve was present in 2 of 12 patients with an isolated PLC injury. LaPrade and Terry ${ }^{18)}$ and Krukhaug et al. ${ }^{19)}$ reported that in patients with posterolateral knee injuries, including combined injuries, peroneal nerve injuries were observed in $13 \%$ of 71 patients and in $16 \%$ of 25 patients, respectively. Patients with chronic injuries complain of broad pain such as medial joint line pain, lateral joint line pain, and posterolateral pain $^{20,21)}$. Patients may also have common peroneal nerve injuries and present with paresthesia or numbness as well. They often show functional instability when the knee is in extension, such as knee giving way into hyperextension during activities like walking down and up the stairs ${ }^{22)}$.

\section{2) Mechanism of injury}

Injuries to the posterolateral structures of the knee are commonly caused by sports injuries, falls, and vehicle accidents. The mechanism of posterolateral injuries can be described in various aspects. A direct hit on the proximal tibia when the knee is in stretched condition may cause an isolated posterolateral injury. Combined hyperextension and varus forces on the knee can also cause an injury to the posterolateral ligaments. In addition, posterior stress force can cause the injury when the knee is in flexed condition or the tibia is in externally rotated position. Lateral dislocation of the knee joint can cause a severe injury to the posterolateral structures.

\section{Clinical Evaluation}

1) Symptoms and signs

Symptoms of the posterolateral injury include a wide range of oppressive pain, ecchymosis, edema, and hardening. Moreover, it is advised to note the patient's lower extremity alignment in standing and walking.

\section{(1) Standing}

Patients with a posterolateral injury are likely to show unusual alignment of the lower extremity. In standing position, they may present with a varus alignment of the $\mathrm{knee}^{23,24)}$.

\section{(2) Gait}

When the static stabilizers of the knee are injured, the dynamic stabilizers cannot function properly due to the convexity of the lateral condyle of the femur and the lateral plateau of the tibia. This causes a varus thrust gait in the stance phase, resulting in abnormal gait ${ }^{25,26)}$. The varus thrust of the knee is seen during the loading-response phase of gait in the presence of a chronic posterolateral knee injury (Fig. 2). Usually, the gait pattern is accompanied by a lift-off of the lateral compartment of the knee, which increases medial compartment joint stress and consequently results in wear of the medial compartment cartilage if untreated ${ }^{27)}$. Sometimes patients show fixed knee gait resulting from adaption to the instability of the knee joint. 


\section{2) Clinical tests}

\section{(1) Dial test}

The dial test is one of the most important physical examinations used to diagnose injury of the posterolateral structures. With the patient positioned in prone position, external rotation of the tibia and thigh-foot angle are assessed. This test is conducted in $30^{\circ}$ and $90^{\circ}$ of knee flexion (Fig. 3). In the case of an isolated PCL injury, more than $10^{\circ}$ of external rotation of the injured knee is present at $30^{\circ}$ of flexion, but not at $90^{\circ}$ of flexion. When a PCL

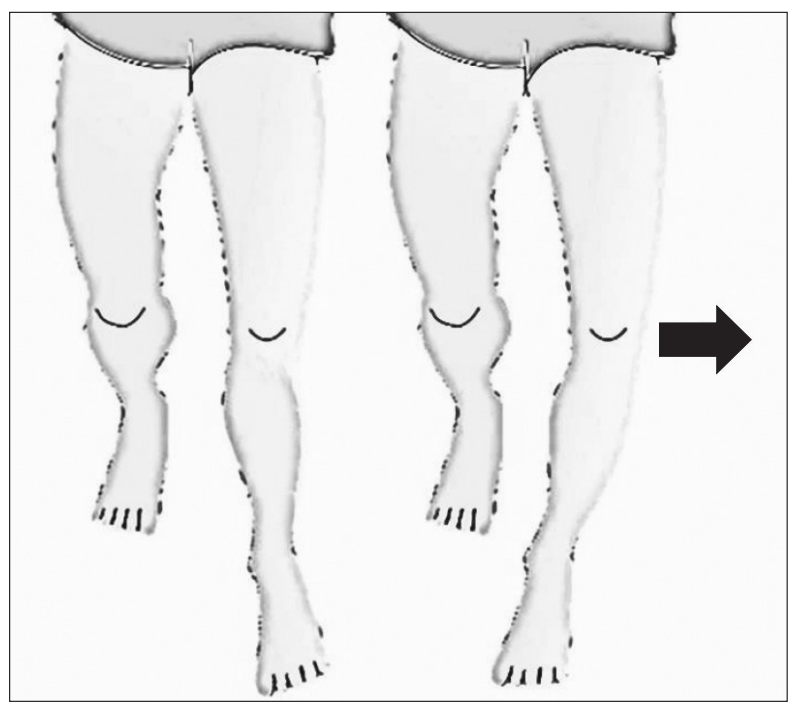

Fig. 2. The left illustration shows normal gait during the loading-response phase of gait. The right illustration shows varus thrust gait during weight bearing on the injured knee. injury is combined, more than $10^{\circ}$ of external rotation in the injured knee is present at both $30^{\circ}$ and $90^{\circ}$ of flexion.

\section{(2) External rotation recurvatum test}

The external rotation recurvatum test may be used to evaluate posterolateral rotatory instability. Hughston et al. ${ }^{20)}$ described this test as a diagnostic tool for posterolateral rotatory instability in the extended knee. The test is performed by lifting the patiente is perform in the supine position, while maintaining the knee in full extension. The extent of extension, tibial rotation, and varus degree are assessed by comparing with the contralateral knee. The knee with a PLC injury will fall into relative hyperextension laterally, and the tibia will be externally rotated into relative varus ${ }^{28)}$. When accompanied by a PCL injury, these positive signs are more prominent.

\section{(3) Posterolateral drawer test}

This test is conducted by applying posterolateral force on the proximal tibia with the hip flexed to $45^{\circ}$, the knee flexed to $90^{\circ}$ and the tibia rotated $15^{\circ}$ externally in supine position. When the tibial condyle shows more external rotation than the lateral femoral condyle, it indicates the presence of a posterolateral injury.

\section{(4) Posterolateral external rotation test}

The posterolateral external rotation test is a combination of the dial test and the posterolateral drawer test. Posterolateral subluxation of the tibia is checked under the simultaneous application of posterior and external rotation forces on the knee joint. Subluxation at $30^{\circ}$ of flexion, but not at $90^{\circ}$ of flexion, indicates the
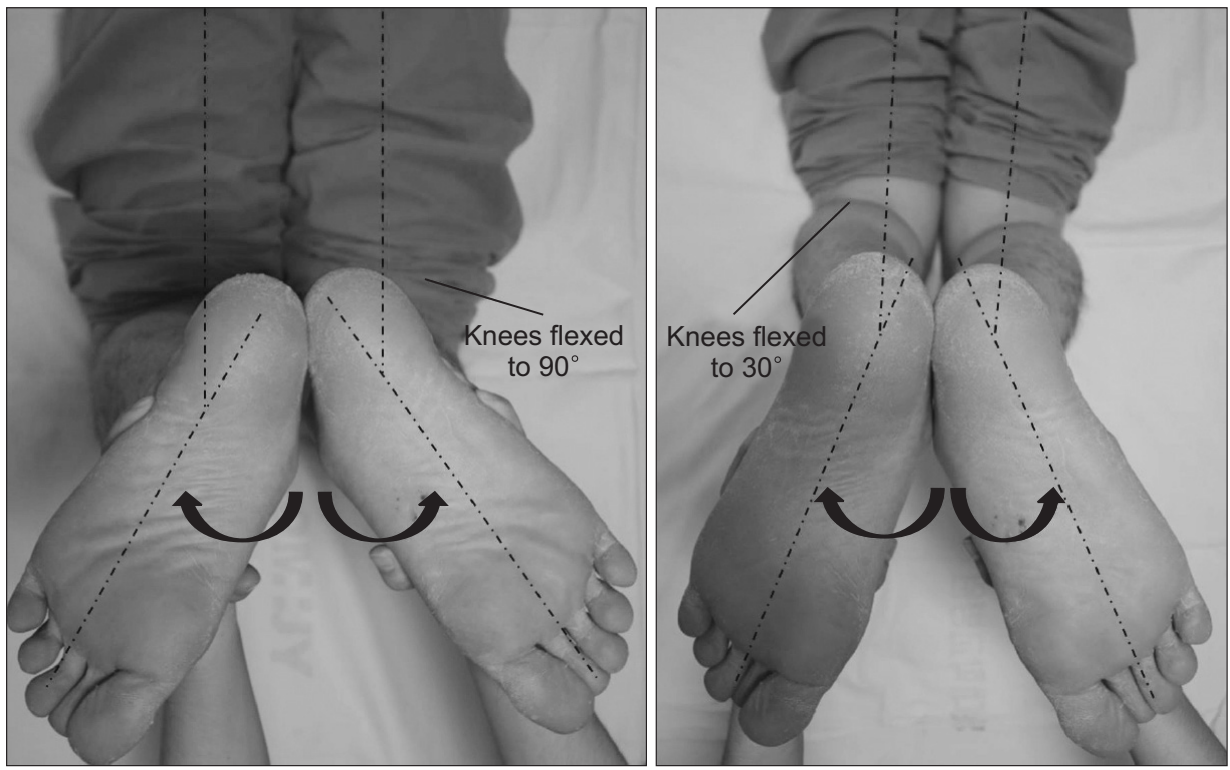

Fig. 3. Dial test performed with the patient in prone position with the knees flexed to $90^{\circ}$ and $30^{\circ}$. The knees are flexed to $90^{\circ}$ in the left photograph and $30^{\circ}$ in the right photograph. 
presence of an isolated posterolateral injury. When a PCL injury is combined, subluxation occurs at both $30^{\circ}$ and $90^{\circ}$ of flexion.

\section{(5) Reverse pivot shift test}

This test is performed with the knee flexed to $40^{\circ}$ and the tibia in external rotation. As the knee is extended, the tibia is reduced with a clicking sound. This indicates the presence of a PLC injury. However, the test has a false positive rate of up to $35 \%$ when performed under anesthesia.

\section{(6) Varus stress test}

The varus stress test at $20^{\circ}$ to $30^{\circ}$ of knee flexion helps to diagnose posterolateral instability of the knee. When the LCL is intact, no increase in varus gapping is seen with the knee is at $20^{\circ}$ to $30^{\circ}$ of flexion. When other structural injuries to the popliteus tendon or PFL are combined, increased varus gapping may be observed. The leg is placed over the examining table with the knee flexed between $20^{\circ}$ and $30^{\circ}$. The examiner's fingers are placed over the joint line stabilizing the distal femur. Then a varus stress is loaded on the knee. To determine the amount of instability, varus gapping is assessed on the stress radiograph.
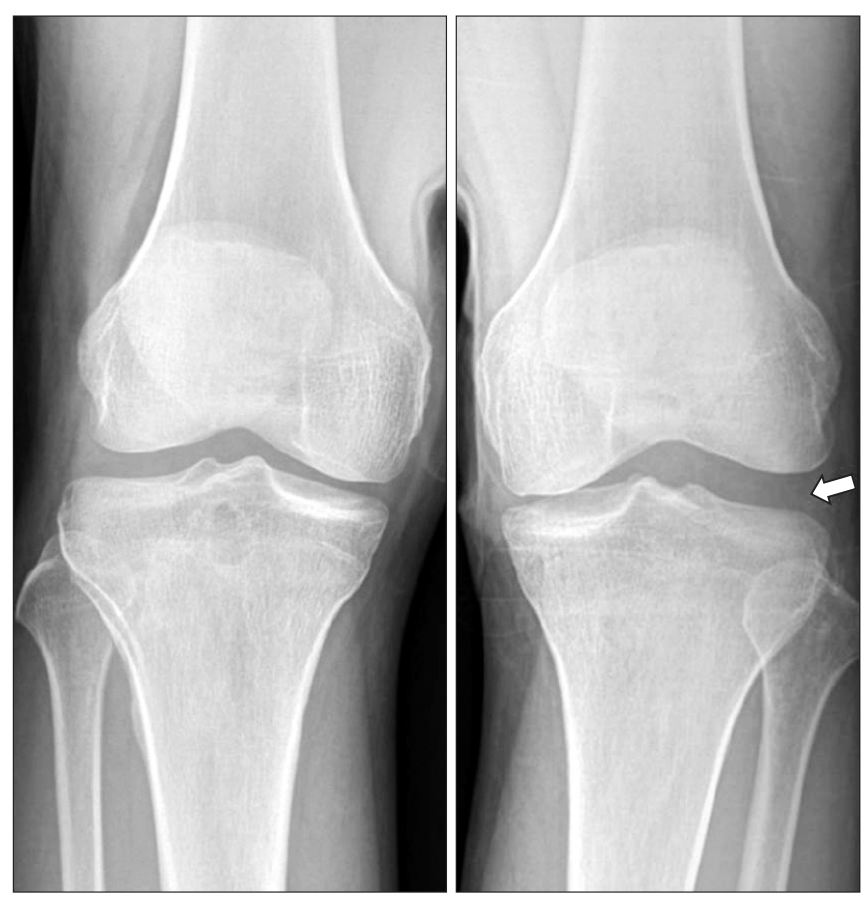

Fig. 4. Varus stress radiographs demonstrating increased opening at the lateral joint line. The right radiograph shows a $14 \mathrm{~mm}$ opening (arrow) in the injured knee.

\section{Imaging}

1) Plain radiography

Plain radiography with anteroposterior (AP), lateral, and axial views is taken to rule out other injuries such as fractures. Lateral joint space widening or tibial metaphysical avulsion fracture such as fibular head avulsion fracture can be seen on the AP view ${ }^{23)}$.

A standing long leg AP view may be taken in the case of chronic injuries for limb alignment correction. The limb alignment should be corrected by an osteotomy prior to or during a reconstruction procedure ${ }^{29)}$.

\section{2) Stress radiography}

Varus stress and kneeling PCL stress radiographs are very helpful in the diagnosis of PLC injuries. LaPrade et al. ${ }^{30)}$ assessed varus stress radiographs with the knee at $20^{\circ}$ of flexion to provide objective measures of lateral compartment gapping (Fig. 4). They reported that an increased opening of more than $4 \mathrm{~mm}$ may indicate a grade III PLC injury. In addition, the kneeling PCL stress radiograph also facilitates objective quantification of isolated or combined PLC injuries $^{31)}$ (Table 1).

\section{3) Magnetic resonance imaging (MRI)}

MRI test can be beneficial when an injury of the posterolateral structures is difficult to diagnose clinically. It helps to identify PLC structures. Especially, the T2-weighted coronal oblique view is more useful in the evaluation of the posterolateral structures than the traditional coronal or sagittal view. MRI is also helpful to evaluate acute or subacute PLC injuries (Fig. 5). Therefore, MRI should be taken within 12 weeks. It has been reported that only about $26 \%$ of the patients can be diagnosed when an MRI is taken after 12 weeks ${ }^{32)}$.

Table 1. Instability Evaluation Using Stress Radiography

\begin{tabular}{cl}
\hline \multicolumn{1}{c}{ Variable } & \multicolumn{1}{c}{ Degree of injury } \\
\hline Varus stress radiograph $(\mathrm{mm})$ & \\
$<2.7$ & Normal knee or minor sprains \\
$2.7-4$ & Complete LCL tear \\
$>4$ & Complete posterolateral injury \\
PCL stress radiograph $(\mathrm{mm})$ & \\
$<4$ & Variation in normal knee or minor \\
& sprains \\
$4-12$ & Isolated PCL injuries \\
$>12$ & Combined injuries of PCL \& PLC
\end{tabular}

LCL: lateral collateral ligament, PCL: posterior cruciate ligament, PLC: posterolateral corner. 

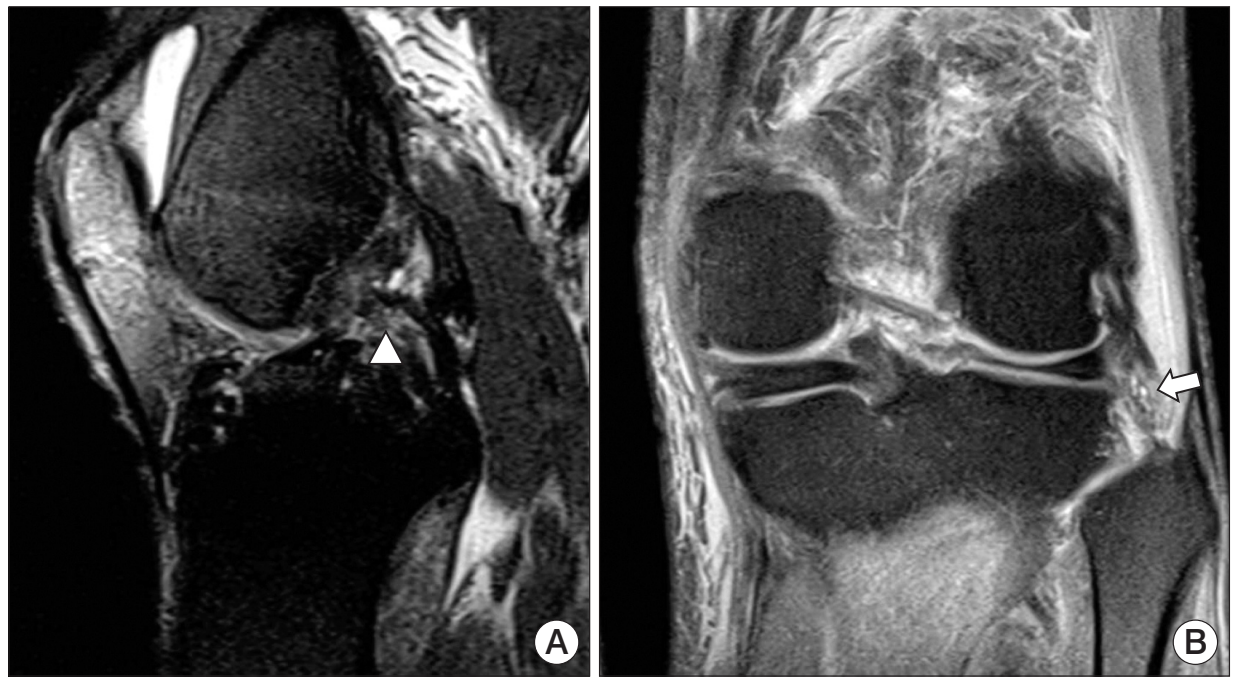

Fig. 5. Sagittal and coronal magnetic resonance imaging scans of a posterolateral corner injury in the left knee. (A) Discontinuity of the posterior cruciate ligament (arrow head) is seen in the sagittal view. (B) High signal on the lateral collateral ligament (arrow) is seen in the coronal view.

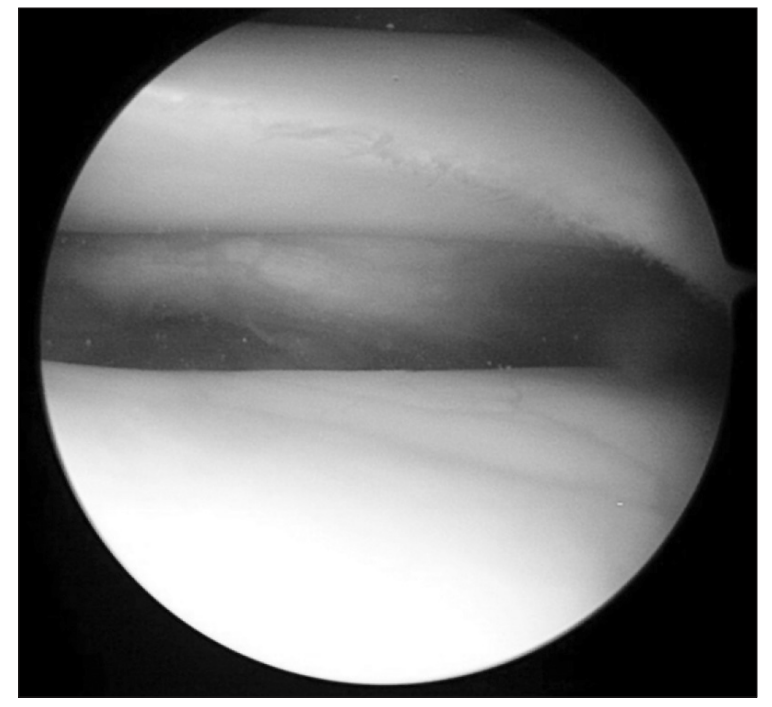

Fig. 6. Lateral joint opening (drive-through sign) observed in arthroscopy.

\section{Arthroscopy}

Arthroscopy provides intraarticular information of posterolateral structures, such as the popliteus complex, coronary ligament of the lateral meniscus, and posterolateral capsule. It helps to decide the appropriate treatment and provides accurate anatomical information in surgical treatment.

A drive-through sign occurs when there is more than $1 \mathrm{~cm}$ lateral joint opening under varus stress to the knee joint, which can be confirmed with arthroscopy (Fig. 6). Also, popliteal hiatus widening during internal rotation of the tibia, tears of the inferior and superior popliteomeniscal fascicle, and abnormal popliteomeniscal motion during rotation may be observed in arthroscopy $^{33)}$.
Table 2. Classification of Damage in Posterolateral Structures

\begin{tabular}{ccc}
\hline Classification & \multicolumn{1}{c}{ Scale of damage } & Damaged structure \\
\hline Type A & $\begin{array}{c}10^{\circ} \text { increase in external rotation } \\
\text { of the tibia }\end{array}$ & PFL, popliteus tendon \\
Type B & $\begin{array}{c}10^{\circ} \text { increase in external rotation } \\
\text { of the tibia } \\
\text { Slight varus relaxation (5-10 } \\
\text { mm increase in varus load test) }\end{array}$ & PFL, popliteus tendon \\
& LCL \\
Type C & $\begin{array}{c}10^{\circ} \text { increase in external rotation } \\
\text { of the tibia }\end{array}$ & PFL, popliteus tendon \\
& $\begin{array}{c}\text { Severe varus relaxation (>10 } \\
\text { mm increase in varus load }\end{array}$ & LCL, capsule avulsion, \\
test) & cruciate ligament
\end{tabular}

PFL: popliteofibular ligament, LCL: lateral collateral ligament.

\section{Classification}

PLC injuries can be classified according to the damage to the posterolateral structures or the degree of posterolateral instability. The following two classifications are most commonly used.

Bleday et al. ${ }^{34)}$ and Fanelli and Larson ${ }^{35)}$ classified the PLC injuries into type $\mathrm{A}, \mathrm{B}$, and $\mathrm{C}$ based on damage to structures (Table 2). Type A injuries involve the PFL and popliteus tendon. Clinically, only an increase in tibial external rotation is observed. Type $B$ injuries affect the PFL, popliteus tendon, and LCL. Mild varus opening is observed in the varus stress test at $30^{\circ}$ of knee flexion along with an increase in tibial external rotation. Type $\mathrm{C}$ injuries involve the PFL, popliteus tendon, LCL, lateral capsular avulsion, and cruciate ligament disruption. Marked varus instability is seen in type $\mathrm{C}$ injuries at $30^{\circ}$ of knee flexion.

The Hughston classification, is based on the assessment of 
varus instability or rotational instability under varus stress force with the knee in full extension ${ }^{20,28)}$ (Table 3). Grade I represents minimal tearing of a ligament with no abnormal motion. Grade II injury shows partial tearing with slight or moderate abnormal motion. Grade Ш injury refers to complete tearing with marked abnormal movements. Despite subjectivity and lack of relation to anatomic cutting studies, this classification method is still important in determining treatment choices.

\section{Treatment}

\section{Non-Operative Treatment}

Grade I and grade II isolated PLC injuries can be treated with non-operative management. Despite the lack of the reports on the outcome of non-operative treatment in the literature, the reported outcomes of non-operative treatment for grade I and II injuries were good. Minimal radiographic changes were reported at 8-year follow-up in patients with an early mobilization proto$\mathrm{col}^{19,36)}$. DeLeo et al. ${ }^{37)}$ reported a case of an 18-year-old female who had a grade II LCL sprain in combination with a PLC injury.

Table 3. Classification of Posterolateral Instability Assessed by Varus or Rotational Instability

\begin{tabular}{cll}
\hline Classification & \multicolumn{1}{c}{ Varus or Rotational instability } & PCL injury \\
\hline Grade I & $0-5 \mathrm{~mm}$ or $0^{\circ}-5^{\circ}$ & Intact PCL \\
Grade II & $5-10 \mathrm{~mm}$ or $6^{\circ}-10^{\circ}$ & Intact PCL \\
Grade III & $>10 \mathrm{~mm}$ or $>10^{\circ}$ (soft endpoint) & PCL rupture \\
\hline
\end{tabular}

PCL: posterior cruciate ligament.
The patient returned to previous level of activity without surgical treatment after only rehabilitation. Appropriate rehabilitation and gait training may be helpful in treating grade I or grade II injuries. Non-operative treatment may offer good outcomes; however, care should be taken considering non-operative treatment of complete tears involving the PLC has shown poor functional results $^{38)}$.

\section{Operative Treatment}

For grade III and grade II PLC injuries accompanied by other structural injuries, surgical management is recommended (Fig. 7).

\section{1) Acute PLC injury}

The choice of treatment depends on the time of injury. In general, an acute injury is defined as an injury that happened within 3 weeks prior to treatment. Acute injuries can be treated with direct repair or augmentation. If the grade of injury is severe or tissue is not vital enough, augmentation or reconstruction can be considered instead of primary repair. The hamstring tendon, biceps tendon, and ITB are candidates for allograft. When reconstructing the posterolateral structures, anatomic reconstruction is more recommendable than non-anatomic reconstruction. Advancement procedures can also be considered. However, such procedures are non-anatomical and non-isometric, increasing the risk of consequently limiting the knee joint movement and leading to failure. It is vital to diagnose all the accompanying injuries. When treating avulsion injuries, firm fixation or suture is necessary.

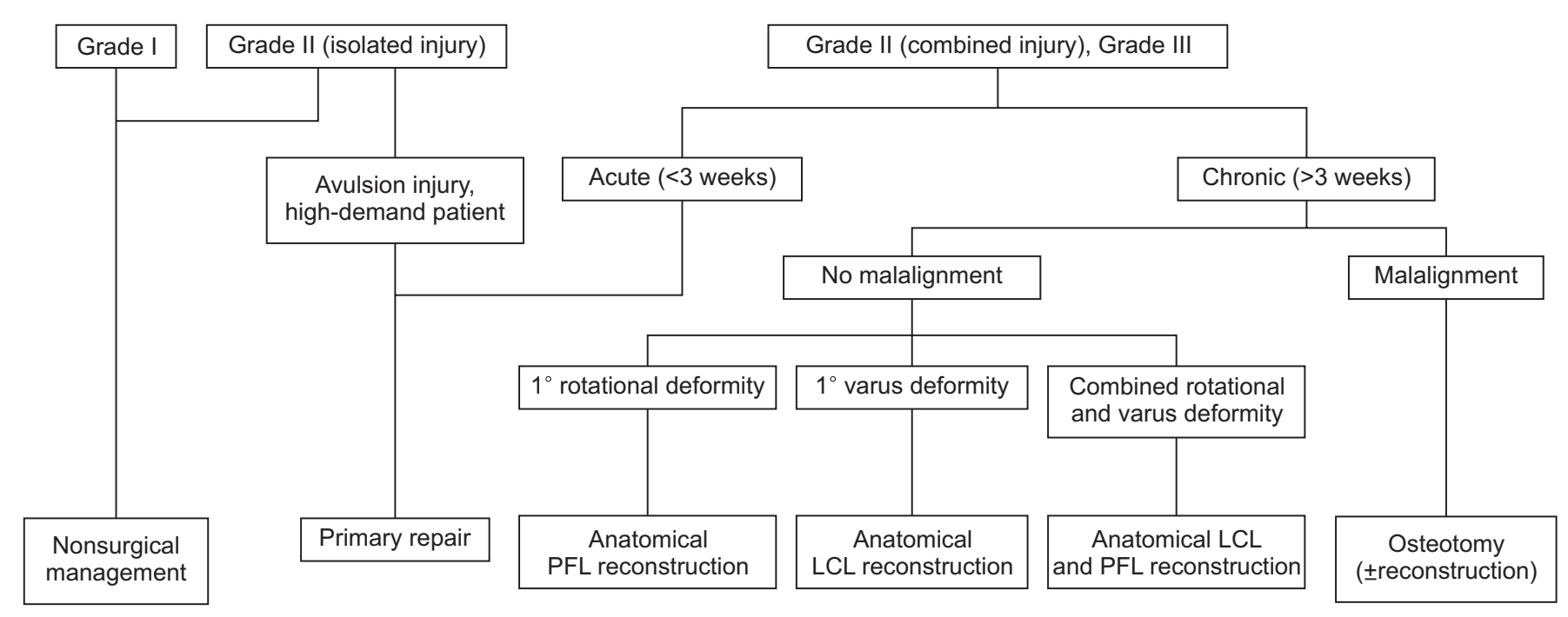

Fig. 7. Algorithm of posterolateral corner treatment according to the chronic or acute injury. PFL: popliteofibular ligament, LCL: lateral collateral ligament. 


\section{2) Chronic PLC injury}

A chronic injury of the posterolateral structure is one that has persisted for more than 3 weeks following injury. The torn structure becomes fibrotic scar tissue after 3 weeks and direct repair can be difficult owing to tissue adhesion. Moreover, joint stiffness may occur after surgery. Therefore, reconstruction is recommended in chronic injuries. Also, the alignment of lower extremity and gait patterns are considerably important in chronic cases. Since the limb alignment is the most important factor to consider in lower limb reconstructive surgery, diagnosis and treatment of limb malalignment should not be overlooked in the management of chronic ligamentous instability ${ }^{39)}$. If there is more than $3^{\circ}$ of varus deviation or the hip-knee axis passes within $30 \%$ of the medial side of the tibial plateau, high tibial osteotomy can be considered.

\section{3) Reconstruction}

Reconstruction of the posterolateral structures is suitable for chronic instability rather than acute cases. There are various methods of reconstruction, which can be divided into anatomic reconstruction and non-anatomic reconstruction.

\section{(1) Non-anatomic reconstruction}

Non-anatomic reconstruction is to obtain posterolateral stability by applying tension on the uninjured posterolateral structures. Arcuate complex or bone block advancement, extracapsular ITB sling, augmentation technique, and bicep tenodesis are recommended for non-anatomic reconstruction. In 2003, Kim et al. ${ }^{40)}$ reported altered biceps tenodesis as a single reconstruction method for posterolateral structures. The average postoperative Lysholm score was 93.6 in the study.

\section{(2) Anatomic reconstruction}

Anatomical and biomechanical research of posterolateral structures has been conducted recently and precise anatomic reconstruction of the injured LCL, popliteus tendon and PFL is recommended with use of the fibular-based technique and tibiofibularbased technique.

In 2005, Larsen et al. ${ }^{41)}$ reported fibular sling. It is a fibularbased technique that can make the popliteal complex and LCL balanced appropriately. This method is commonly used because it is quite a simple procedure that provides good results. Camarda et al. ${ }^{42)}$ reported that the fibular-based technique offered excellent results in chronic posterolateral instability patients whose average postoperative Lysholm knee score was 94 . Ho et al. ${ }^{43}$ conducted a cadaver study comparing the results between the non-anatomic reconstruction group and the anatomic reconstruction group using the fibular-based technique: the anatomic reconstruction group obtained better results. Niki et al. ${ }^{44)}$ reported excellent clinical results of a modified Larson's procedure (Fig. 8) and emphasized the recovery of tension in the PFL and LCL. For the recovery, the article underlined the importance of fixation in the fibular tunnel. In 2014, Kuzma et al. ${ }^{45)}$ reported anatomic reconstruction of the PFL and LCL using the fibular-based technique and the Achilles tendon as an allograft. With this method, the reconstructed ligament can be repaired with the existing popliteus
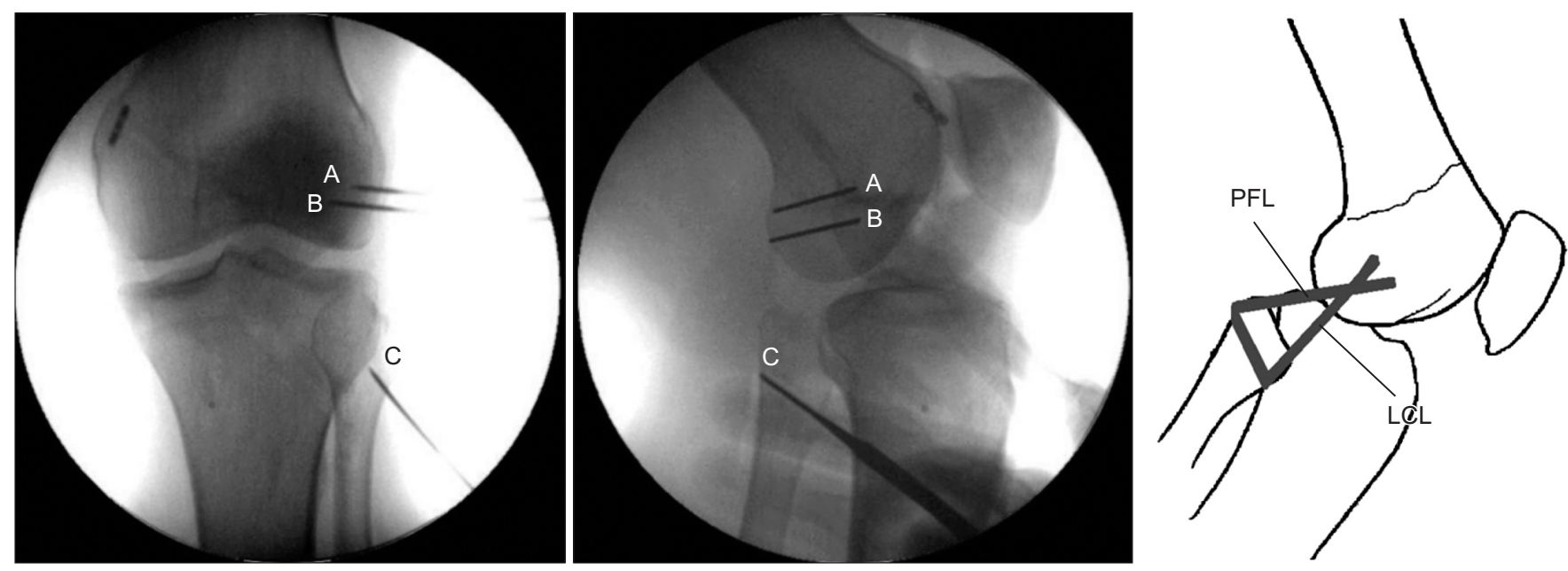

Fig. 8. Radiographs showing the fixation points of allograft through the fibular tunnel as described in the illustration (modified larson technique). A, B: femoral tunnel. Anatomical insertions of LCL and popliteus tendon at the lateral femoral condyle aimed toward the flare of the medial femoral epicondyle, C: fibular tunnel. Starting point is set at the distal anterolateral portion of the fibular head (insertion of LCL) exiting proximal posteromedial portion of the fibular head (insertion of PFL). LCL: lateral collateral ligament, PFL: popliteofibular ligament. 
tendon. In addition, this technique does not require a transtibial tunnel.

LaPrade and Wentorf ${ }^{9}$ introduced the tibiofibular-based technique to reconstruct all of the LCLs, popliteal tendon, and PFL, which are the important structures of the PLC. Yoon et al. ${ }^{46)}$ and Lee et al. ${ }^{47)}$ reported a tibiofibular-based technique using the Achilles tendon as an allograft (Fig. 9). The varus and external rotation were reduced significantly in the anatomic reconstruction group compared to the non-anatomic reconstruction group. The tibiofibular-based technique seems to be advantageous since it allows for anatomic reconstruction of the three important structures. However, this method is somewhat difficult to perform and may excessively limit the posterolateral motion. Yoon et al. ${ }^{15)}$ reported that there was no significant difference between the group that had all three structures reconstructed and the group where the popliteus tendon was not reconstructed.

There has been few long-term research on the PLC reconstruction; however, the short-term studies showed good results ${ }^{48)}$. Many cadaveric studies have compared the fibular-based technique with the tibiofibular-based technique. However, the results have shown little conformity. Kim et al. ${ }^{16)}$ compared the operation methods that reconstruct only two structures among three structures in a cadaveric model. The results showed no significant difference among the methods and none was effective for restoring normal function of the knee joint. McCarthy et al. ${ }^{49)}$ compared reconstruction of all three components with reconstruction of only the popliteal tendon and LCL. The results were better after reconstruction of all three components. Thus, they recom-

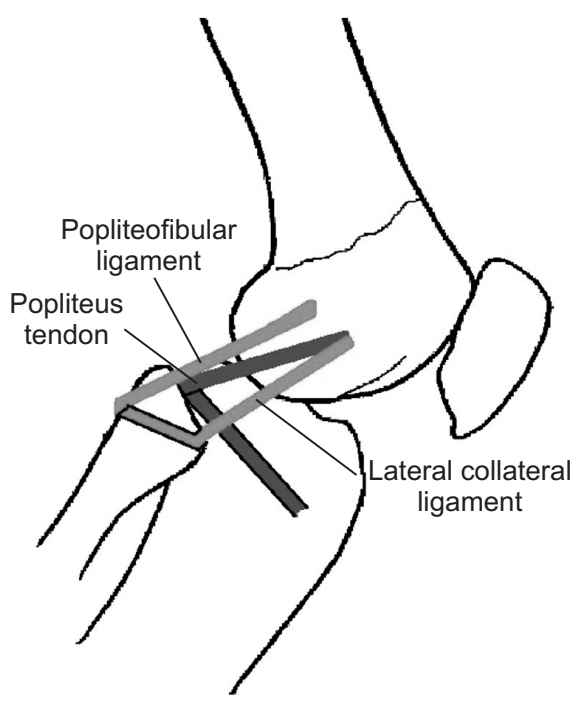

Fig. 9. Achilles tendon was split into two bundles and anatomic double bundle reconstruction was performed. mended reconstruction of all of the three structures. Miyatake et al. ${ }^{50)}$ conducted a comparison study between the two-strand reconstruction and the four-strand reconstruction the four-strand reconstruction provided better biomechanical results.

\section{Postoperative Rehabilitation}

The purpose of postoperative rehabilitation is to protect the reconstructed or repaired ligament structures. Strengthening of the quadriceps muscle and protection of the patellofemoral joint are emphasized in early rehabilitation. This gradually leads to muscle strengthening, functional exercises, and daily activities so that the patient may eventually participate in sports activities.

Many studies follow the guidelines of Robert F. LaPrade for posterolateral reconstruction involving the popliteus tendon, PFL, and LCL. However, guidelines may be modified depending on other associated knee ligament injuries. After PLC reconstruction, the patient should stay immobilized and non-weight bearing for 6 weeks ${ }^{1,4}$. During the immobilization period, the patient may wear an immobilizer brace with the knee extended except for range of motion exercises ${ }^{29)}$. Rehabilitation begins immediately after surgery and initial rehabilitation focuses on restoring tibiofemoral and patellofemoral range of motion. For the first 2 weeks, passive range of motion exercises are performed from $0^{\circ}$ to $90^{\circ}$, which then progresses to full range of motion as tolerated ${ }^{4)}$. At 6 weeks, patients are permitted to begin spinning on a stationary bike and wean off crutches. After full weight bearing, the exercise is focused on developing muscular endurance. An exercise such as closed chain strengthening is permitted. Then, the exercise focuses on progressive muscular strength development. Isolated hamstring strengthening is limited to avoid stress on reconstruction until a minimum of 4 postoperative month ${ }^{1,4,29}$. Running or agility training may begin once appropriate strength and power are restored. At approximately 6 months after surgery, return to sports activities is allowed after checking the muscle strength, stability of the joint, and range of motion.

\section{Clinical Outcomes}

Treatment outcomes of PLC injuries can vary according to the severity of injury, associated ligament pathologies, and the treatment of choice.

\section{Outcomes of Acute Repair}

Outcomes of repair of acute PLC injuries are better when performed earlier in the acute stage. Shelbourne et al. ${ }^{51)}$ reported that 
repair of the PLC by 4 weeks post-injury resulted in significantly better outcome than repair performed between 4 to 6 weeks postinjury. The better outcomes of early repair may be associated with management of the problems presented by tissue retraction, adhesion, and scarring of the peroneal nerve that may occur in the first few weeks after injury. If the surgery is performed within the first 2 weeks of injury, the anatomy is much easier to identify and anatomic repair can often be achieved with ease ${ }^{52)}$.

Baker et al. ${ }^{53)}$ reported a follow-up series of 13 patients who underwent PLC repair. They had good objective and functional outcomes. Krukhaug et al. ${ }^{19)}$ retrospectively reviewed patients who had been treated with acute repair or conservative treatment. Patients who had acute repair showed improvement in instability of the knee, while the conservatively treated patients showed no improvement.

However, recent studies have reported that primary repair is not sufficient to treat injuries of the LCL, popliteus tendon, or $\mathrm{PFL}^{38,54,55)}$.

\section{Outcomes of Reconstruction}

Acute reconstruction is thought to be an effective treatment option for PLC injuries with irreparable soft tissue. Ibrahim et al reported the outcomes of 20 patients who underwent acute bicruciate reconstruction and PLC reconstruction using the contralateral hamstring as an autograft ${ }^{56)}$. The study showed improved outcome scores after acute reconstruction: the mean Lysholm score was 90 points at the 44-month follow-up. Stannard et al. ${ }^{54)}$ found a higher failure rate in the repair group than in the reconstruction group. Levy et al. ${ }^{55)}$ reported cases of multiligament knee injury patients who underwent repair, followed by delayed reconstruction of the cruciate ligaments. The failure rate was much lower in them than in the repair only group.

In recent studies, reconstruction is described to have better outcomes. However, outcomes may differ according to the choice of different reconstruction methods the surgeons make. A handful of studies reported improved patient outcomes using an anatomic technique. Stannard et al. ${ }^{57)}$ reported outcomes of 15 patients who underwent anatomic reconstruction: the mean Lysholm knee score was 92. LaPrade et al. ${ }^{48)}$ demonstrated an anatomic PLC reconstruction technique in 2004 and reported outcomes of a cohort group afterwards: the patients showed significant improvement in IKDC objective scores after surgery.

\section{Complications}

\section{Persistent Instability}

Instability may continue after repair or reconstruction surgery. Technical errors may result in persistent instability if the major anatomic structures are not restored ${ }^{10,58)}$. The LCL, popliteus, and PFL should be restored with either reconstruction or repair ${ }^{59)}$.

Also, instability may differ according to the treatment of choice. Stannard at al. ${ }^{54)}$ and Levy et al. ${ }^{55)}$ examined failure rates of PLC repairs and reconstructions. Failure rates of repairs were higher than those of reconstructions in the studies. To prevent persistent instability after surgery, it is vital to choose an appropriate surgical treatment according to the indications.

Varus malalignment of the knee can be another factor in persistent laxity ${ }^{59)}$. A staged approach may be necessary in chronic PLC injuries or failure of previous reconstruction.

\section{Neurovascular Problems}

Common peroneal nerve injury may be accompanied by PLC injuries owing to its close proximity ${ }^{60)}$. When the knee is subjected to varus and hyperextension forces associated with a PLC injury, the common peroneal nerve is vulnerable ${ }^{61)}$. Therefore, special caution is necessary during surgery.

Deep vein thrombosis (DVT) may occur after any lower extremity surgery. Therefore, DVT may also occur after PLC reconstruction or repair. In high risk patients, there should be a prophylactic administration of low molecular weight heparin if necessary. Early mobilization and rehabilitation is also effective for preventing DVT.

\section{Infection after Surgery}

Superficial or deep infection has always been a potential problem in all knee surgeries. In open knee reconstruction, the incidence of wound infection is thought to range from $0.3 \%$ to $12.5 \%{ }^{62,63)}$. Prophylactic antibiotics, meticulous soft tissue handling, and careful planning of skin incision may help reduce wound problems ${ }^{59)}$.

Posterolateral reconstruction is preferred to direct repair in surgical treatment of PLC injuries. Reconstruction of posterolateral structures is required in the chronic stage, and anatomic reconstruction is more recommended than non-anatomic reconstruction. There are two types of techniques for anatomic reconstruction: fibular-based technique and tibiofibular-based technique. Currently, fibular-based reconstruction is preferred to the tibiofibular-based technique. This may be because the comparable biomechanical performance and technical ease of 
the fibula-based reconstruction coupled with preservation of the remaining tissue provide advantages that tip the balance in favor of this approach.

\section{Conflict of Interest}

No potential conflict of interest relevant to this article was reported.

\section{References}

1. Lunden JB, Bzdusek PJ, Monson JK, Malcomson KW, Laprade RF. Current concepts in the recognition and treatment of posterolateral corner injuries of the knee. J Orthop Sports Phys Ther. 2010;40:502-16.

2. Sanchez AR 2nd, Sugalski MT, LaPrade RF. Anatomy and biomechanics of the lateral side of the knee. Sports Med Arthrosc. 2006;14:2-11.

3. Terry GC, LaPrade RF. The posterolateral aspect of the knee: anatomy and surgical approach. Am J Sports Med. 1996;24: 732-9.

4. Chahla J, Moatshe G, Dean CS, LaPrade RF. Posterolateral corner of the knee: current concepts. Arch Bone Jt Surg. 2016;4:97-103.

5. Gollehon DL, Torzilli PA, Warren RF. The role of the posterolateral and cruciate ligaments in the stability of the human knee: a biomechanical study. J Bone Joint Surg Am. 1987;69:233-42.

6. Grood ES, Stowers SF, Noyes FR. Limits of movement in the human knee. Effect of sectioning the posterior cruciate ligament and posterolateral structures. J Bone Joint Surg Am. 1988;70:88-97.

7. Shahane SA, Ibbotson C, Strachan R, Bickerstaff DR. The popliteofibular ligament: an anatomical study of the posterolateral corner of the knee. J Bone Joint Surg Br. 1999;81:63642.

8. Veltri DM, Deng XH, Torzilli PA, Warren RF, Maynard MJ. The role of the cruciate and posterolateral ligaments in stability of the knee: a biomechanical study. Am J Sports Med. 1995;23:436-43.

9. LaPrade RF, Wentorf F. Diagnosis and treatment of posterolateral knee injuries. Clin Orthop Relat Res. 2002;(402):11021.

10. Harner CD, Hoher J. Evaluation and treatment of posterior cruciate ligament injuries. Am J Sports Med. 1998;26:471-82.

11. LaPrade RF, Wozniczka JK, Stellmaker MP, Wijdicks CA.
Analysis of the static function of the popliteus tendon and evaluation of an anatomic reconstruction: the "fifth ligament" of the knee. Am J Sports Med. 2010;38:543-9.

12. Harner CD, Vogrin TM, Höher J, Ma CB, Woo SL. Biomechanical analysis of a posterior cruciate ligament reconstruction. Deficiency of the posterolateral structures as a cause of graft failure. Am J Sports Med. 2000;28:32-9.

13. Sekiya JK, Haemmerle MJ, Stabile KJ, Vogrin TM, Harner $\mathrm{CD}$. Biomechanical analysis of a combined double-bundle posterior cruciate ligament and posterolateral corner reconstruction. Am J Sports Med. 2005;33:360-9.

14. LaPrade RF, Johansen S, Wentorf FA, Engebretsen L, Esterberg JL, Tso A. An analysis of an anatomical posterolateral knee reconstruction: an in vitro biomechanical study and development of a surgical technique. Am J Sports Med. 2004;32:1405-14.

15. Yoon KH, Lee JH, Bae DK, Song SJ, Chung KY, Park YW. Comparison of clinical results of anatomic posterolateral corner reconstruction for posterolateral rotatory instability of the knee with or without popliteal tendon reconstruction. Am J Sports Med. 2011;39:2421-8.

16. Kim SJ, Kim HS, Moon HK, Chang WH, Kim SG, Chun YM. A biomechanical comparison of 3 reconstruction techniques for posterolateral instability of the knee in a cadaveric model. Arthroscopy. 2010;26:335-41.

17. DeLee JC, Riley MB, Rockwood CA Jr. Acute posterolateral rotatory instability of the knee. Am J Sports Med. 1983;11: 199-207.

18. LaPrade RF, Terry GC. Injuries to the posterolateral aspect of the knee. Association of anatomic injury patterns with clinical instability. Am J Sports Med. 1997;25:433-8.

19. Krukhaug Y, Mølster A, Rodt A, Strand T. Lateral ligament injuries of the knee. Knee Surg Sports Traumatol Arthrosc. 1998;6:21-5.

20. Hughston JC, Andrews JR, Cross MJ, Moschi A. Classification of knee ligament instabilities: part II. The lateral compartment. J Bone Joint Surg Am. 1976;58:173-9.

21. Hughston JC, Jacobson KE. Chronic posterolateral rotatory instability of the knee. J Bone Joint Surg Am. 1985;67:351-9.

22. Covey DC. Injuries of the posterolateral corner of the knee. J Bone Joint Surg Am. 2001;83:106-18.

23. Bae WH, Ha JK, Kim JG. Treatment of posterolateral rotatory instability of the knee. J Korean Knee Soc. 2010;22:1-10.

24. Towne LC, Blazina ME, Marmor L, Lawrence JF. Lateral compartment syndrome of the knee. Clin Orthop Relat Res. 1971;76:160-8. 
25. Ricchetti ET, Sennett BJ, Huffman GR. Acute and chronic management of posterolateral corner injuries of the knee. Orthopedics. 2008;31:479-88.

26. Noyes FR, Grood ES, Torzilli PA. Current concepts review: the definitions of terms for motion and position of the knee and injuries of the ligaments. J Bone Joint Surg Am. 1989; 71:465-72.

27. Sharma L, Song J, Felson DT, Cahue S, Shamiyeh E, Dunlop DD. The role of knee alignment in disease progression and functional decline in knee osteoarthritis. JAMA. 2001;286: 188-95.

28. Ranawat A, Baker CL 3rd, Henry S, Harner CD. Posterolateral corner injury of the knee: evaluation and management. J Am Acad Orthop Surg. 2008;16:506-18.

29. Crespo B, James EW, Metsavaht L, LaPrade RF. Injuries to posterolateral corner of the knee: a comprehensive review from anatomy to surgical treatment. Rev Bras Ortop. 2014; 50:363-70

30. LaPrade RF, Heikes C, Bakker AJ, Jakobsen RB. The reproducibility and repeatability of varus stress radiographs in the assessment of isolated fibular collateral ligament and gradeIII posterolateral knee injuries: an in vitro biomechanical study. J Bone Joint Surg Am. 2008;90:2069-76.

31. Jackman T, LaPrade RF, Pontinen T, Lender PA. Intraobserver and interobserver reliability of the kneeling technique of stress radiography for the evaluation of posterior knee laxity. Am J Sports Med. 2008;36:1571-6.

32. Pacheco RJ, Ayre CA, Bollen SR. Posterolateral corner injuries of the knee: a serious injury commonly missed. J Bone Joint Surg Br. 2011;93:194-7.

33. Kim JG, Moon HT, Hwang IH, Kim JH, Song JK. Arthroscopic evaluation of posterolateral rotatory instability of the knee. J Korean Orthop Assoc. 2003;38:29-33.

34. Bleday RM, Fanelli GC, Giannotti BF, Edson CJ, Barrett TA. Instrumented measurement of the posterolateral corner. Arthroscopy. 1998;14:489-94.

35. Fanelli GC, Larson RV. Practical management of posterolateral instability of the knee. Arthroscopy. 2002;18(2 Suppl 1): 1-8.

36. Kannus P. Nonoperative treatment of grade II and III sprains of the lateral ligament compartment of the knee. Am J Sports Med. 1989;17:83-8.

37. DeLeo AT, Woodzell WW, Snyder-Mackler L. Resident's case problem: diagnosis and treatment of posterolateral instability in a patient with lateral collateral ligament sprain. J Orthop Sports Phys Ther. 2003;33:185-91.
38. Geeslin AG, LaPrade RF. Outcomes of treatment of acute grade-III isolated and combined posterolateral knee injuries: a prospective case series and surgical technique. J Bone Joint Surg Am. 2011;93:1672-83.

39. Savarese E, Bisicchia S, Romeo R, Amendola A. Role of high tibial osteotomy in chronic injuries of posterior cruciate ligament and posterolateral corner. J Orthop Traumatol. 2011; 12:1-17.

40. Kim SJ, Shin SJ, Jeong JH. Posterolateral rotatory instability treated by a modified biceps rerouting technique: technical considerations and results in cases with and without posterior cruciate ligament insufficiency. Arthroscopy. 2003;19:4939.

41. Larsen MW, Moinfar AR, Moorman CT 3rd. Posterolateral corner reconstruction: fibular-based technique. J Knee Surg. 2005;18:163-6.

42. Camarda L, Condello V, Madonna V, Cortese F, D’Arienzo M, Zorzi C. Results of isolated posterolateral corner reconstruction. J Orthop Traumatol. 2010;11:73-9.

43. Ho EP, Lam MH, Chung MM, Fong DT, Law BK, Yung PS, Chan WY, Chan KM. Comparison of 2 surgical techniques for reconstructing posterolateral corner of the knee: a cadaveric study evaluated by navigation system. Arthroscopy. 2011;27:89-96.

44. Niki Y, Matsumoto H, Otani T, Enomoto H, Toyama Y, Suda Y. A modified Larson's method of posterolateral corner reconstruction of the knee reproducing the physiological tensioning pattern of the lateral collateral and popliteofibular ligaments. Sports Med Arthrosc Rehabil Ther Technol. 2012; $4: 21$.

45. Kuzma SA, Chow RM, Engasser WM, Stuart MJ, Levy BA. Reconstruction of the posterolateral corner of the knee with achilles tendon allograft. Arthrosc Tech. 2014;3:e393-8.

46. Yoon KH, Bae DK, Ha JH, Park SW. Anatomic reconstructive surgery for posterolateral instability of the knee. Arthroscopy. 2006;22:159-65.

47. Lee KH, Jung YB, Jung HJ, Jang EC, Song KS, Kim JY, Lee $\mathrm{SH}$. Combined posterolateral corner reconstruction with remnant tensioning and augmentation in chronic posterior cruciate ligament injuries: minimum 2-year follow-up. Arthroscopy. 2011;27:507-15.

48. LaPrade RF, Johansen S, Agel J, Risberg MA, Moksnes H, Engebretsen L. Outcomes of an anatomic posterolateral knee reconstruction. J Bone Joint Surg Am. 2010;92:16-22.

49. McCarthy M, Camarda L, Wijdicks CA, Johansen S, Engebretsen L, Laprade RF. Anatomic posterolateral knee recon- 
structions require a popliteofibular ligament reconstruction through a tibial tunnel. Am J Sports Med. 2010;38:1674-81.

50. Miyatake S, Kondo E, Tsai TY, Hirschmann M, Halewood C, Jakobsen BW, Yasuda K, Amis AA. Biomechanical comparisons between 4-strand and modified Larson 2-strand procedures for reconstruction of the posterolateral corner of the knee. Am J Sports Med. 2011;39:1462-9.

51. Shelbourne KD, Haro MS, Gray T. Knee dislocation with lateral side injury: results of an en masse surgical repair technique of the lateral side. Am J Sports Med. 2007;35:1105-16.

52. Spitzer E, Doyle JB, Marx RG. Outcomes of surgical treatment of posterolateral instability of the knee. J Knee Surg. 2015;28:471-4.

53. Baker CL Jr, Norwood LA, Hughston JC. Acute combined posterior cruciate and posterolateral instability of the knee. Am J Sports Med. 1984;12:204-8.

54. Stannard JP, Brown SL, Farris RC, McGwin G Jr, Volgas DA. The posterolateral corner of the knee: repair versus reconstruction. Am J Sports Med. 2005;33:881-8.

55. Levy BA, Dajani KA, Morgan JA, Shah JP, Dahm DL, Stuart MJ. Repair versus reconstruction of the fibular collateral ligament and posterolateral corner in the multiligamentinjured knee. Am J Sports Med. 2010;38:804-9.
56. Ibrahim SA, Ghafar S, Salah M, Abo Alnas M, Al Misfer A, Farouk H, Al Haran H, Khirait S. Surgical management of traumatic knee dislocation with posterolateral corner injury. Arthroscopy. 2013;29:733-41.

57. Stannard JP, Brown SL, Robinson JT, McGwin G Jr, Volgas DA. Reconstruction of the posterolateral corner of the knee. Arthroscopy. 2005;21:1051-9.

58. Fanelli GC, Giannotti BF, Edson CJ. The posterior cruciate ligament arthroscopic evaluation and treatment. Arthroscopy. 1994;10:673-88.

59. MacDonald P, Vo A. Complications of posterolateral corner injuries of the knee and how to avoid them. Sports Med Arthrosc. 2015;23:51-4.

60. Deutsch A, Wyzykowski RJ, Victoroff BN. Evaluation of the anatomy of the common peroneal nerve: defining nerve-atrisk in arthroscopically assisted lateral meniscus repair. Am J Sports Med. 1999;27:10-5.

61. White J. The results of traction injuries to the common peroneal nerve. J Bone Joint Surg Br. 1968;50:346-50.

62. Graf B, Uhr F. Complications of intra-articular anterior cruciate reconstruction. Clin Sports Med. 1988;7:835-48.

63. Hughston JC. Complications of anterior cruciate ligament surgery. Orthop Clin North Am. 1985;16:237-40. 